\title{
Perturbation theory of computing QCD jet cross sections beyond NLO accuracy
}

\author{
Zoltán Trócsányi ${ }^{* \dagger}$ \\ University of Debrecen and Institute of Nuclear Research of the Hungarian Academy of Sciences \\ H-4001 Debrecen P.O.Box 51, Hungary \\ E-mail: Zoltan.Trocsanyi@cern.ch
}

\section{Gábor Somogyi}

University of Zürich

Winterthurerstrasse 190, CH-8057 Zürich, Switzerland

E-mail: sgabi@physik.unizh.ch

We discuss the problems that arise when one wishes to extend the existing general methods of computing radiative corrections to QCD jet cross sections to beyond next-to-leading order. Then we present a subtraction scheme that can be defined at any order in perturbation theory. We give a status report of the implementation of the method to computing jet cross sections in electronpositron annihilation at the next-to-next-to-leading order accuracy.

8th International Symposium on Radiative Corrections

October 1-5, 2007

Florence, Italy

\footnotetext{
* Speaker.

$\dagger$ I thank the Galileo Galilei Institute for Theoretical Physics for the hospitality during this conference. This research was supported by the Hungarian Scientific Research Fund grant OTKA K-60432 and by the Swiss National Science Foundation (SNF) under contract 200020-117602.
} 
Accurate predictions of $\mathrm{QCD}$ jet cross sections require the computation of radiative corrections at least at next-to-leading order (NLO) accuracy, but in some cases also at higher order. The physical cases when computations at the next-to-next-to-leading order (NNLO) are important have been discussed extensively in the literature [1]. There are also some less standard motivations. Although several general methods exist for computations at NLO, these become very time-consuming with increasing number of final-state partons. Many talks at this conference showed impressive progress in computing one-loop corrections to amplitudes of multileg processes [2], therefore, we should consider the question wether we can compute the real radiation corrections fast enough. Furthermore, the thorough understanding of computing NNLO corrections may help the combination of parton showers and NLO calculations.

The perturbative expansion of any jet cross section can formally be written as $\sigma=\sigma^{\mathrm{LO}}+$ $\sigma^{\mathrm{NLO}}+\sigma^{\mathrm{NNLO}}+\ldots$. Let us consider $e^{+} e^{-} \rightarrow m$ jet production, when $\sigma^{\mathrm{LO}}$ is the integral of the fully exclusive Born cross section over the available phase space defined by the jet function $J_{m}$,

$$
\sigma^{\mathrm{LO}}=\int_{m} \mathrm{~d} \sigma_{m}^{\mathrm{B}} J_{m} \equiv \int \mathrm{d} \phi_{m}\left|\mathscr{M}_{m}^{(0)}\right|^{2} J_{m}
$$

There are two contributions to the NLO correction. We have to consider the fully exclusive cross section $\mathrm{d} \sigma^{\mathrm{R}}$ for producing $m+1$ partons and the one-loop correction $\mathrm{d} \sigma^{\mathrm{V}}$ to the production of $m$ partons,

$$
\sigma^{\mathrm{NLO}}=\int_{m+1} \mathrm{~d} \sigma^{\mathrm{R}} J_{m+1}+\int_{m} \mathrm{~d} \sigma^{\mathrm{V}} J_{m}=\int \mathrm{d} \phi_{m+1}\left|\mathscr{M}_{m+1}^{(0)}\right|^{2} J_{m+1}+\int \mathrm{d} \phi_{m} 2 \operatorname{Re}\left\langle\mathscr{M}_{m}^{(1)} \mid \mathscr{M}_{m}^{(0)}\right\rangle J_{m} .
$$

These two contributions are separately divergent in $d=4$ dimensions although their sum is finite for infrared safe observables. We assume that ultraviolet renormalization has been carried out, so the divergences are purely of infrared origin and are regularized by defining the integrals in $d=4-2 \varepsilon$ dimensions.

There are several general methods of computing the finite NLO correction. Most of these rely on the same principles, namely one defines approximate cross section $\mathrm{d} \sigma^{\mathrm{A}}$ which regularizes the real correction in $d$ dimensions in all its infrared singular limits that lead to poles in $\varepsilon$, so the cross section

$$
\sigma_{m+1}^{\mathrm{NLO}}=\int_{m+1}\left[\left(\mathrm{~d} \sigma^{\mathrm{R}}\right)_{\varepsilon=0} J_{m+1}-\left(\mathrm{d} \sigma^{\mathrm{A}}\right)_{\varepsilon=0} J_{m}\right]
$$

is finite. ${ }^{1}$ The approximate cross section is constructed using the universal soft- and collinear factorization properties of QCD matrix elements (we use the colour-state notation [3] and also some notation introduced in Ref. [4]), ${ }^{2}$

$$
\mathrm{S}_{r}\left|\mathscr{M}_{m+1}^{(0)}\left(p_{r}, \ldots\right)\right|^{2} \propto \sum_{\substack{i, k \\ i \neq k}} \frac{s_{i k}}{s_{i r} s_{k r}}\left\langle\mathscr{M}_{m}^{(0)}(\ldots)\left|T_{i} \cdot T_{k}\right| \mathscr{M}_{m}^{(0)}(\ldots)\right\rangle
$$

\footnotetext{
${ }^{1}$ The subtraction terms in these equations are symbolic in the sense that these are actually sums of different terms. The jet function depends on different momenta in each of these terms, the exact set of momenta for each term can be found in Ref. [18].

${ }^{2}$ We drop some numerical factors in order to keep the expressions as simple as possible, as only the structure of these formulae is relevant for the discussion.
} 


$$
\mathrm{C}_{i r}\left|\mathscr{M}_{m+1}^{(0)}\left(p_{i}, p_{r}, \ldots\right)\right|^{2} \propto \frac{1}{s_{i r}}\left\langle\mathscr{M}_{m}^{(0)}\left(p_{i r}, \ldots\right)\left|\hat{P}_{i r}^{(0)}\right| \mathscr{M}_{m}^{(0)}\left(p_{i r}, \ldots\right)\right\rangle .
$$

These factorization formulae allow for such a construction that the integration over the phase space of the unresolved parton can be computed independently of the jet function, leading to

$$
\int_{1} \mathrm{~d} \sigma^{\mathrm{A}}=\mathrm{d} \sigma_{m}^{\mathrm{B}} \otimes I(\varepsilon)
$$

where $I(\varepsilon)$ is an operator in the colour space with universal pole part,

$$
I(\varepsilon) \propto \frac{\alpha_{\mathrm{s}}}{2 \pi} \sum_{i}\left[\frac{1}{\varepsilon} \gamma_{i}-\frac{1}{\varepsilon^{2}} \sum_{k \neq i} T_{i} \cdot T_{k}\left(\frac{4 \pi \mu^{2}}{s_{i k}}\right)^{\varepsilon}\right]+\mathrm{O}\left(\varepsilon^{0}\right) .
$$

This pole part is equal, but opposite in sign to the pole part of the virtual correction, so that the $m$-parton integral

$$
\sigma_{m}^{\mathrm{NLO}}=\int_{m}\left[\mathrm{~d} \sigma^{\mathrm{V}}+\int_{1} \mathrm{~d} \sigma^{\mathrm{A}}\right]_{\varepsilon=0}
$$

is finite. Therefore, the sum of the two finite contributions $\sigma_{m}^{\mathrm{NLO}}$ and $\sigma_{m+1}^{\mathrm{NLO}}$ is equal to $\sigma^{\mathrm{NLO}}$.

In constructing the approximate cross section special care is needed to avoid double subtractions in the regions where a soft parton becomes also collinear to another hard parton. At the NLO accuracy, the overlap of the soft- and collinear limits can easily be identified to be the collinear limit of the soft factorization formula [4]. However, disentangling the multiple unresolved limits at higher orders, when multiple soft-, collinear- and soft-collinear limits overlap in a complicated way, is far more cumbersome [4]. This calls for a simple and systematic procedure.

In a physical gauge the collinear singularities are due to the collinear splitting of an external parton [5]. The overall colour structure of the event does not change, the splitting is entirely described by the Altarelli-Parisi functions which are a product of colour factors and a kinematical function describing the collinear kinematics of the splitting. Thus, if we want to identify the collinear contributions in the soft factorization formulae to any order in perturbation theory, we can use the following simple procedure: (i) employ the soft insertion rules [6,7] to obtain the usual expression

$$
\mathrm{S}_{r}\left|\mathscr{M}_{m+1}^{(0)}\left(p_{r}, \ldots\right)\right|^{2} \propto \sum_{i=1}^{m} \sum_{k=1 \text { hel. }}^{m} \varepsilon_{\mu}\left(p_{r}\right) \varepsilon_{v}^{*}\left(p_{r}\right) \frac{2 p_{i}^{\mu} p_{k}^{v}}{s_{i r} s_{k r}}\left\langle\mathscr{M}_{m}^{(0)}(\ldots)\left|T_{i} \cdot T_{k}\right| \mathscr{M}_{m}^{(0)}(\ldots)\right\rangle,
$$

with

$$
s_{i r}=2 p_{i} \cdot p_{r} \quad \text { and } \quad \sum_{\text {hel. }} \varepsilon_{\mu}\left(p_{r}\right) \varepsilon_{v}^{*}\left(p_{r}\right)=-g^{\mu v}+\frac{p_{r}^{\mu} n^{v}+p_{r}^{v} n^{\mu}}{p_{r} \cdot n}
$$

(ii) choose Coulomb gauge $\left(n^{\mu}=Q^{\mu}-p_{r}^{\mu} Q^{2} / s_{r Q}, s_{r Q}=2 p_{r} \cdot p_{Q}\right)$ to identify the collinear contribution in the colour diagonal terms

$$
\begin{aligned}
\mathrm{S}_{r}\left|\mathscr{M}_{m+1}^{(0)}\left(p_{r}, \ldots\right)\right|^{2} \propto \sum_{i=1}^{m} & {\left[\frac{1}{2} \sum_{k \neq i}^{m}\left(\frac{s_{i k}}{s_{i r} s_{r k}}-\frac{2 s_{i Q}}{s_{r Q} s_{i r}}-\frac{2 s_{k Q}}{s_{r Q} s_{k r}}\right)\left\langle\mathscr{M}_{m}^{(0)}(\ldots)\left|T_{i} \cdot T_{k}\right| \mathscr{M}_{m}^{(0)}(\ldots)\right\rangle\right.} \\
& \left.-T_{i}^{2} \frac{2}{s_{i r}} \frac{s_{i Q}}{s_{r Q}}\left|\mathscr{M}_{m}^{(0)}(\ldots)\right|^{2}\right]
\end{aligned}
$$


(iii) define momentum fractions in the Sudakov parametrization of momenta $p_{i}^{\mu}$ and $p_{r}^{\mu}$ being collinear as $z_{i}=\frac{s_{i Q}}{s_{i Q}+s_{r Q}}$, so that the colour-diagonal terms become equal to the collinear limit of the soft factorization formula. Then the pure soft contributions are given by

$$
\mathrm{S}_{r}^{\text {pure }}\left|\mathscr{M}_{m+1}^{(0)}\left(p_{r}, \ldots\right)\right|^{2} \propto \sum_{i=1}^{m}\left[\frac{1}{2} \sum_{k \neq i}^{m}\left(\frac{s_{i k}}{s_{i r} s_{r k}}-\frac{2 s_{i Q}}{s_{r Q} s_{i r}}-\frac{2 s_{k Q}}{s_{r Q} s_{k r}}\right)\left\langle\mathscr{M}_{m}^{(0)}(\ldots)\left|T_{i} \cdot T_{k}\right| \mathscr{M}_{m}^{(0)}(\ldots)\right\rangle\right] .
$$

We checked explicitly that this procedure leads to non-overlapping factorization formulae that describe the analytic behaviour of the squared matrix elements in any IR limit at the NNLO accuracy [8]. Furthermore, the factorization formula in the purely soft limit is independent of the helicity of the soft gluon. This allows for the definition of approximate cross sections for real radiation with fixed helicities, and thus for Monte Carlo summation over the helicities in NLO computations. The Monte Carlo summation over the helicities in computations at LO was found very useful for saving CPU time [9].

The physical motivation for higher accuracy and the success of the subtraction schemes at NLO lead one to consider the extension of the subtraction method to NNLO, when three terms contribute: the double-real, the real-virtual and the double-virtual cross sections,

$$
\sigma^{\mathrm{NNLO}}=\sigma_{m+2}^{\mathrm{RR}}+\sigma_{m+1}^{\mathrm{RV}}+\sigma_{m}^{\mathrm{VV}} \equiv \int_{m+2} \mathrm{~d} \sigma_{m+2}^{\mathrm{RR}} J_{m+2}+\int_{m+1} \mathrm{~d} \sigma_{m+1}^{\mathrm{RV}} J_{m+1}+\int_{m} \mathrm{~d} \sigma_{m}^{\mathrm{VV}} J_{m} .
$$

The necessary ingredients for constructing approximate cross sections, namely (i) the tree level three-parton splitting functions [10] and double soft $g g$ and $q \bar{q}$ currents [7, 11] and (ii) the one-loop two-parton splitting functions [12] and soft-gluon current [13] that is, the infrared (IR) structures of the three contributions at NNLO have been known for some time. The difficulty of using the multiple infrared factorization formulae for cunstructing the approximate cross sections is amply demonstrated by the slow progress in setting up a subtraction scheme. Other approaches to computing NNLO corrections have been more successful.

The antennae subtraction method [14] uses complete squared matrix elements instead of the IR structure and the first complete computation of NNLO corrections to three-jet production in electron-positron annihilation has been reported to this conference [15]. For processes involving massive particles and/or simple kinematics, direct numerical evaluation of the coefficients in the Laurent expansion of the three contributions (based on sector decomposition) lead to the complete NNLO corrections of Higgs- [16] and vector-boson production in hadron collisions [17]

The reorganization of the NNLO contributions into three finite cross sections,

$$
\sigma^{\mathrm{NNLO}}=\sigma_{m+2}^{\mathrm{NNL}}+\sigma_{m+1}^{\mathrm{NNLO}}+\sigma_{m}^{\mathrm{NNLO}}
$$

is governed by the jet function as follows:

$$
\begin{gathered}
\sigma_{m+2}^{\mathrm{NNLO}}=\int_{m+2}\left\{\mathrm{~d} \sigma_{m+2}^{\mathrm{RR}} J_{m+2}-\mathrm{d} \sigma_{m+2}^{\mathrm{RR}, \mathrm{A}_{2}} J_{m}-\left(\mathrm{d} \sigma_{m+2}^{\mathrm{RR}, \mathrm{A}_{1}} J_{m+1}-\mathrm{d} \sigma_{m+2}^{\mathrm{RR}, \mathrm{A}_{12}} J_{m}\right)\right\} \\
\sigma_{m+1}^{\mathrm{NNLO}}=\int_{m+1}\left\{\left(\mathrm{~d} \sigma_{m+1}^{\mathrm{RV}}+\int_{1} \mathrm{~d} \sigma_{m+2}^{\mathrm{RR}, \mathrm{A}_{1}}\right) J_{m+1}-\left[\mathrm{d} \sigma_{m+1}^{\mathrm{RV}, \mathrm{A}_{1}}+\left(\int_{1} \mathrm{~d} \sigma_{m+2}^{\mathrm{RR}, \mathrm{A}_{1}}\right) \mathrm{A}_{1}\right] J_{m}\right\}
\end{gathered}
$$

and

$$
\sigma_{m}^{\mathrm{NNLO}}=\int_{m}\left\{\mathrm{~d} \sigma_{m}^{\mathrm{VV}}+\int_{2}\left(\mathrm{~d} \sigma_{m+2}^{\mathrm{RR}, \mathrm{A}_{2}}-\mathrm{d} \sigma_{m+2}^{\mathrm{RR}, \mathrm{A}_{12}}\right)+\int_{1}\left[\mathrm{~d} \sigma_{m+1}^{\mathrm{RV}, \mathrm{A}_{1}}+\left(\int_{1} \mathrm{~d} \sigma_{m+2}^{\mathrm{RR}, \mathrm{A}_{1}}\right)^{\mathrm{A}_{1}}\right]\right\} J_{m} .
$$


Let us concentrate on Eq. (16). The construction of $\mathrm{d} \sigma_{m+1}^{\mathrm{RV}, \mathrm{A}_{1}}$ that regularizes the kinematical singularities of $\mathrm{d} \sigma_{m+1}^{\mathrm{RV}}$ in the singly-unresolved regions is straightforward, but the difference $\left[\mathrm{d} \sigma_{m+1}^{\mathrm{RV}} J_{m+1}-\mathrm{d} \sigma_{m+1}^{\mathrm{RV}, \mathrm{A}_{1}} J_{m}\right]_{\varepsilon=0}$ is infinite. To make it finite, we have to subtract the universal pole part, given by Eqs. (6)-(8), too. The latter however, does not obey universal collinear factorization. Due to coherent soft-gluon emission from unresolved partons only the sum $\left\langle\mathscr{M}_{m+1}^{(0)}\right|\left(T_{j} \cdot T_{k}+T_{r}\right.$. $\left.T_{k}\right)\left|\mathscr{M}_{m+1}^{(0)}\right\rangle$ factorizes in the collinear limit $\left(T_{j r}=T_{j}+T_{r}\right)$,

$$
\mathrm{C}_{j r}\left\langle\mathscr{M}_{m+1}^{(0)}\left|\left(T_{j} \cdot T_{k}+T_{r} \cdot T_{k}\right)\right| \mathscr{M}_{m+1}^{(0)}\right\rangle \propto \frac{1}{s_{j r}}\left\langle\mathscr{M}_{m}^{(0)}\left|T_{j r} \cdot T_{k} \hat{P}_{j r}^{(0)}\right| \mathscr{M}_{m}^{(0)}\right\rangle .
$$

This factorization is violated by the factors $s_{i k}^{-\varepsilon} / \varepsilon^{2}$ at $\mathrm{O}\left(\varepsilon^{0}\right)$, which was also noticed in Ref. [19], where it was shown that the terms that violate factorization are known to give vanishing contribution after integration. However, if one insists on defining fully local subtractions, which is important for numerical stability and reducing CPU time, then the use of properly defined new approximate cross sections is necessary. The complete subtraction scheme at NNLO, based on these new approximate cross sections is defined in Refs. $[18,20]$. We employed this subtraction scheme for computing the finite cross sections $\sigma_{m+2}^{\mathrm{NNLO}}$ and $\sigma_{m+1}^{\mathrm{NNO}}$ of the C-parameter and thrust distributions in electron-positron annihilation. In order to have the complete physical prediction we also have to compute $\sigma_{m}^{\mathrm{NNLO}}$, which requires the integration of the subtraction terms over the singlyand doubly-unresolved factorized phase spaces. We used standard techniques fractioning, sector decomposition [21] and residuum subtraction to find the Laurent expansion of the one-particle integrals in

$$
\int_{1}\left[\mathrm{~d} \sigma_{m+1}^{\mathrm{RV}, \mathrm{A}_{1}}+\left(\int_{1} \mathrm{~d} \sigma_{m+2}^{\mathrm{RR}, \mathrm{A}_{1}}\right)^{\mathrm{A}_{1}}\right] .
$$

We expect that the same techniques can also be employed for the computation of the coefficients in the $\varepsilon$-expansion of the two-particle integral

$$
\int_{2}\left(\mathrm{~d} \sigma_{m+2}^{\mathrm{RR}, \mathrm{A}_{2}}-\mathrm{d} \sigma_{m+2}^{\mathrm{RR}, \mathrm{A}_{12}}\right) .
$$

This work is in progress.

\section{References}

[1] see for instance E. W. N. Glover, Nucl. Phys. Proc. Suppl. 116, 3 (2003) [arXiv:hep-ph/0211412].

[2] C. Papadopoulos, these proceedings. T. Riemann, these proceedings. T. Binoth, these proceedings. D. Forde, these proceedings. P. Mastrolia, these proceedings. Z. Kunszt, these proceedings.

[3] S. Catani and M. H. Seymour, Nucl. Phys. B 485 (1997) 291 [Erratum-ibid. B 510 (1997) 291] [hep-ph/9605323].

[4] G. Somogyi, Z. Trócsányi and V. Del Duca, JHEP 0506, 024 (2005) [arXiv:hep-ph/0502226].

[5] J. Frenkel and J. C. Taylor, Nucl. Phys. B 116, 185 (1976). D. Amati, R. Petronzio and G. Veneziano, Nucl. Phys. B 146, 29 (1978).

[6] A. Bassetto, M. Ciafaloni and G. Marchesini, Phys. Rept. 100, 201 (1983).

[7] S. Catani and M. Grazzini, Nucl. Phys. B 570, 287 (2000) [arXiv:hep-ph/9908523]. 
[8] Z. Nagy, G. Somogyi and Z. Trócsányi, arXiv:hep-ph/0702273.

[9] P. Draggiotis, R. H. P. Kleiss and C. G. Papadopoulos, Phys. Lett. B 439, 157 (1998) [arXiv:hep-ph/9807207].

[10] A. Gehrmann-De Ridder and E. W. N. Glover, Nucl. Phys. B 517, 269 (1998) [arXiv:hep-ph/9707224]. J. M. Campbell and E. W. N. Glover, Nucl. Phys. B 527 (1998) 264 [arXiv:hep-ph/9710255]. S. Catani and M. Grazzini, Phys. Lett. B 446 (1999) 143 [arXiv:hep-ph/9810389]. D. A. Kosower, Nucl. Phys. B 552, 319 (1999) [arXiv:hep-ph/9901201]. V. Del Duca, A. Frizzo and F. Maltoni, Nucl. Phys. B 568 (2000) 211 [arXiv:hep-ph/9909464]. D. A. Kosower, Phys. Rev. D 67 (2003) 116003 [arXiv:hep-ph/0212097]. D. A. Kosower, Phys. Rev. Lett. 91, 061602 (2003) [arXiv:hep-ph/0301069].

[11] F. A. Berends and W. T. Giele, Nucl. Phys. B 313, 595 (1989).

[12] Z. Bern, L. J. Dixon, D. C. Dunbar and D. A. Kosower, Nucl. Phys. B 425, 217 (1994) [arXiv:hep-ph/9403226].Z. Bern, V. Del Duca and C. R. Schmidt, Phys. Lett. B 445, 168 (1998) [arXiv:hep-ph/9810409]. D. A. Kosower and P. Uwer, Nucl. Phys. B 563, 477 (1999) [arXiv:hep-ph/9903515]. Z. Bern, V. Del Duca, W. B. Kilgore and C. R. Schmidt, Phys. Rev. D 60, 116001 (1999) [arXiv:hep-ph/9903516].

[13] S. Catani and M. Grazzini, Nucl. Phys. B 591 (2000) 435 [arXiv:hep-ph/0007142].

[14] A. Gehrmann-De Ridder, T. Gehrmann and E. W. N. Glover, JHEP 0509, 056 (2005) [arXiv:hep-ph/0505111].

[15] T. Gehrmann, these prceedings.

[16] R. V. Harlander and W. B. Kilgore, Phys. Rev. Lett. 88 (2002) 201801 [arXiv:hep-ph/0201206]. C. Anastasiou and K. Melnikov, Nucl. Phys. B 646, 220 (2002) [arXiv:hep-ph/0207004]. V. Ravindran, J. Smith and W. L. van Neerven, Nucl. Phys. B 665, 325 (2003) [arXiv:hep-ph/0302135]. C. Anastasiou, K. Melnikov and F. Petriello, Phys. Rev. Lett. 93, 262002 (2004) [arXiv:hep-ph/0409088]. C. Anastasiou, K. Melnikov and F. Petriello, Nucl. Phys. B 724, 197 (2005) [arXiv:hep-ph/0501130]. S. Catani and M. Grazzini, Phys. Rev. Lett. 98, 222002 (2007) [arXiv:hep-ph/0703012].

[17] C. Anastasiou, L. J. Dixon, K. Melnikov and F. Petriello, Phys. Rev. Lett. 91 (2003) 182002 [arXiv:hep-ph/0306192]. C. Anastasiou, L. Dixon, K. Melnikov and F. Petriello, Phys. Rev. D 69 (2004) 094008 [arXiv:hep-ph/0312266]. K. Melnikov and F. Petriello, Phys. Rev. Lett. 96, 231803 (2006) [arXiv:hep-ph/0603182]. C. Anastasiou, G. Dissertori and F. Stockli, JHEP 0709, 018 (2007) [arXiv:0707.2373 [hep-ph]].

[18] G. Somogyi, Z. Trócsányi and V. Del Duca, JHEP 0701, 070 (2007) [arXiv:hep-ph/0609042].

[19] A. Gehrmann-De Ridder, T. Gehrmann, E. W. N. Glover and G. Heinrich, JHEP 0711, 058 (2007) [arXiv:0710.0346 [hep-ph]].

[20] G. Somogyi and Z. Trócsányi, Acta Phys. Chim. Debr. XL, 101 (2006) [arXiv:hep-ph/0609041]. G. Somogyi and Z. Trócsányi, JHEP 0701, 052 (2007) [arXiv:hep-ph/0609043].

[21] G. Heinrich, Nucl. Phys. Proc. Suppl. 116 (2003) 368 [arXiv:hep-ph/0211144]. C. Anastasiou, K. Melnikov and F. Petriello, Phys. Rev. D 69, 076010 (2004) [arXiv:hep-ph/0311311]. T. Binoth and G. Heinrich, Nucl. Phys. B 693 (2004) 134 [arXiv:hep-ph/0402265]. G. Heinrich, Nucl. Phys. Proc. Suppl. 135 (2004) 290 [arXiv:hep-ph/0406332]. G. Heinrich, Eur. Phys. J. C 48, 25 (2006) [arXiv:hep-ph/0601062]. C. Bogner and S. Weinzierl, arXiv:0709.4092 [hep-ph]. 